\title{
Modelling the ZalaZONE Proving Ground: a benchmark of State-of-the-art Automotive Simulators PreScan, IPG CarMaker, and VTD Vires
}

\author{
K. Gangel ${ }^{1}$, Z. Hamar ${ }^{1}$, A. Háry ${ }^{2}$, Á. Horváth ${ }^{1}$, G. Jandó ${ }^{1}$, B. \\ Könyves $^{3}$, D. Panker ${ }^{1}$, K. Pintér ${ }^{4}$, M. Pataki ${ }^{1,6}$, M. Szalai ${ }^{6}$, Zs. \\ Szalay ${ }^{1,5}$, T. Tettamanti ${ }^{6 *}$, V. Tihanyi ${ }^{1,5}$, B. Tóth ${ }^{9}$, B. Varga ${ }^{6}$, \\ Zs. J. Viharos ${ }^{7,8}$
}

\section{${ }^{1}$ Automotive Proving Ground Zala Ltd., ZalaZONE Tér 1, H-8900 Zalaegerszeg, Hungary \\ ${ }^{2}$ ZalaZONE Industry Park Ltd. Dr. Michelberger Pál út 3, H-8900 Zalaegerszeg, Hungary \\ ${ }^{3}$ Continental Automotive Hungary Ltd., Házgyári u. 6-8, H-8200 Veszprém, Hungary}

${ }^{4}$ Bay Zoltán Alkalmazott Kutatási Közhasznú Nonprofit Ltd., Kondorfa u. 1, H-1116 Budapest, Hungary

${ }^{5}$ Budapest University of Technology and Economics, Department of Automotive Technologies, Stoczek J. u. 6, H-1111Budapest, Hungary ${ }^{6}$ Budapest University of Technology and Economics, Department of Control for Transportation and Vehicle Systems, Stoczek J. u. 2, H-1111 Budapest, Hungary

${ }^{7}$ Institute for Computer Science and Control, Kende u. 13-17, Budapest $\mathrm{H}-1111$, Hungary

${ }^{8}$ John von Neumann University, Izsáki str. 10, H-6000 Kecskemét, Hungary ${ }^{9}$ TÜV Rheinland - KTI Kft., Than Károly u. 3-5, H-1119 Budapest, Hungary *e-mail: tettamanti@mail.bme.hu

Abstract: In our days, simulation based development is a core element of vehicle engineering, especially considering highly automated or fully autonomous vehicles. Accordingly, the paper presents a benchmark of 
three different automotive simulators: PreScan, IPG CarMaker, and VTD Vires. The three software tools were applied for the same goal, namely, modelling the ZalaZONE Proving Ground of Hungary for vehicle testing. The paper aims to highlight the experiences while creating the virtual models by presenting and comparing the relevant software features and providing suggestions for scientific or practical application.

Keywords: automotive proving ground; simulation software; automotive testing and validation; autonomous vehicles; virtual testing

\section{Introduction}

Autonomous driving became one of the most researched topics of today's vehicle industry. The interest of the society is growing continuously due to the various beneficial effects like the vision of zero accidents, the increasing mobility, and the decreasing pollution, which can change our everyday life as well [1]. However, selfdriving cars have to perform well in those difficult traffic situations where the most developed vehicles mounted with the newest advanced driver assistance systems currently fail. To cover the arising wide range of driving situations, the number of test scenarios and tested kilometres must be increased also continuously [2]. It is already a standard practice in the automotive industry to perform a part of the test kilometres needed for development through simulations. Several virtual testing software tools are available and the number of public datasets is also increasing to create even more realistic scenarios [3]. The software mentioned in the title, IPG CarMaker, VTD Vires, and PreScan are such best practice virtual testing software. These tools are already well known in the automotive industry, providing a variety of realistic and high-precision models for simulations to guarantee real-world testing. The paper reports the applications and comparisons of the experiences on all the mentioned, three software tools for proving ground test simulation.

There are different levels of simulations determined for testing some or all features of vehicles with self-driving capabilities. Typically, numerous scientific research deals with the simulation of vehicle functions on various levels. The lowest level of simulation means testing of generic models and the highest level is the simulation of (almost) all vehicle functions. However, the issue of simulation focusing specifically on proving ground tests has not yet investigated, it is one of the essential contributions of the presented paper.

A related work in [4] provides opportunities for multi-level testing, from Softwarein-the-Loop (SiL) tests to Vehicle-in-the-Loop (ViL) procedures. Many forwardlooking vehicle functions and ADAS (Advanced Driver Assistance System) systems 
have already being tested through simulations, e.g., this study tests a new feature in Active Safety Light in ViL using VTD Vires [5]. ViL testing is also used in the work of [6] by extending the test environment with augmented reality technology to simulate virtual interference in a real-world environment. The contribution in the article [7] reports also on simulations of a Cooperative Collision Warning function. In the case of ADAS systems, the simulation-driven validation process may also play an important role [8], but some companies are also addressing the possibility of virtual validation, e.g., Siemens with PreScan [9]. The article [10] deals with the "mixed virtual and real environment" testing capabilities, in which the virtual test environment also plays a vital role, and a "proof of concept" has been implemented for that concept in [11].

The authors of this article are all experienced in the use of simulation software and are all related to the R\&D works at the ZalaZONE Automotive Proving Ground. 2. ZalaZONE is a unique proving ground for autonomous vehicle technologies constructed near Zalaegerszeg, Hungary (see Section 2. for more details). One of the main goals at ZalaZONE is to facilitate the testing of future vehicles with highly automated and self-driving functions by providing state-of-the-art procedures and technologies to support the execution of vehicle test simulations. Therefore, creating an accurate, virtual model of the complete ZalaZONE Proving Ground was realized in each of the three simulation software mentioned above, which also provides the opportunity to perform tests in this virtual environment, and later in a unique framework created by combining virtual and real-world environments [12]. The paper's goal, in general, is to draw a detailed picture of experiences while creating the ZalaZONE virtual models in these different software tools. It is important to emphasize that this article aims not to prioritize or evaluate any of the software tools but to present and compare their relevant features and serve with advices during their scientific and practical application as well.

The structure of the paper is as follows. Section 2 briefly introduces the ZalaZONE Proving Ground. Section 3 presents the applied simulation software tools, in the next, fourth section, the experiences about the different software environments are provided with particular emphasis on the modelling process and the construction of the test track modules. Conclusions, acknowledgements and references close the paper.

\section{ZalaZONE, a unique proving ground for autonomous vehicle technologies}

The ZalaZONE proving ground is located in Zalaegerszeg, Hungary, Europe. The traditional test track features focusing on driving and driving stability are implemented together with the research and development infrastructure elements for 
future vehicle validation. The proving ground provides not only dynamics tests for conventional vehicles, but it also allows validation tests for autonomous vehicles and electric vehicles as well [13]. The construction works of the ZalaZONE Proving Ground started in 2017 and will be fully completed in 2020 (Fig. 1). ZalaZONE (beyond the classic vehicle dynamics testing) enables to carry out handling and control scenarios, test cutting-edge automotive technologies (such as electric vehicles and self-driving vehicles) as well as communication technologies [14]. The 250 ha area (Fig 2) incorporates the following test features:

- standard vehicle dynamics testing and validation,

- fully integrated autonomous vehicle testing and validation,

- environment preparation (obstacles, traffic signs, traffic control, other vehicles, vulnerable road users),

- complex driving and traffic situations,

- smart city area,

- testing and validation, from prototype testing to mass production.

ZalaZONE Proving Ground enables complex test cycles on either classical or interconnected elements for automated/autonomous vehicles. The main elements of the ZalaZONE test track are listed briefly below.

- Dynamic Platform:

The Dynamic Platform is a special, continuous, asphalt paved element that provides the ability to perform high-speed tests under safe conditions. The dynamic element is a circular surface with a diameter of $300 \mathrm{~m}$, provided with multi-layer asphalt pavement. To the west side of the Dynamic Platform, a $740 \mathrm{~m}$ long acceleration track is connected for safe acceleration during tests.

- Braking Platform:

The braking platform is a track element designed for testing ABS, ASR, and ESP systems with special pavement elements and a built-in irrigation system. This module offers six different lanes with different frictions providing different braking conditions. Moreover, the Braking Platform has the possibility of wetting by lanes and has corresponding drainage. With an acceleration lane of almost $700 \mathrm{~m}$ and a brake surface of $200 \mathrm{~m}$, the Braking Platform makes also possible to test long distance brake tests. 


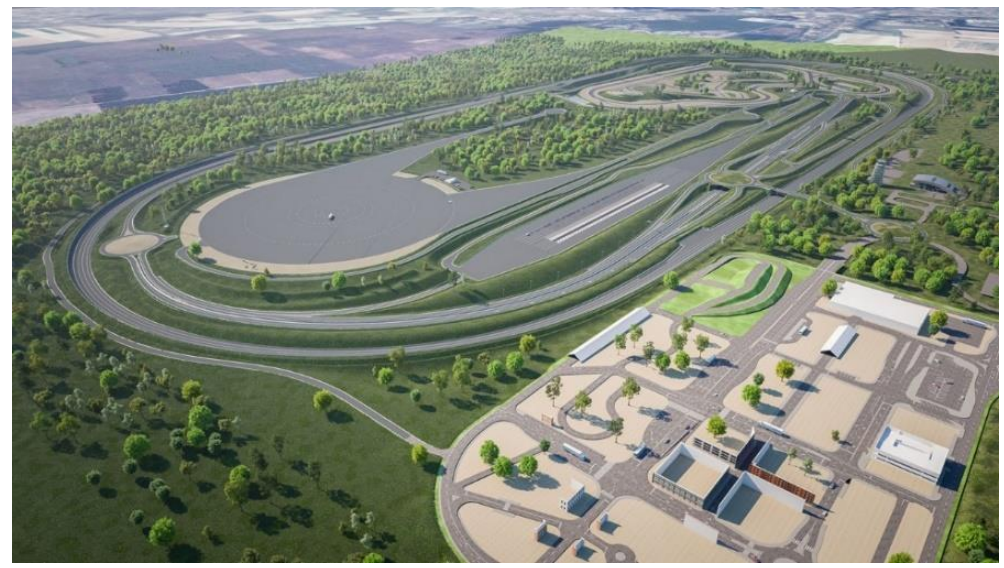

Figure 1. Visualization image of the Automotive proving ground dedicated to autonomous vehicle testing and validation at Zalaegerszeg city, Hungary, Europe (GPS: 46.889771, 16.836956)

- Handling Course:

The Handling Course is a test track element where it is possible to examine vehicle behavior, vehicle handling, and test different technical settings under controlled traffic conditions. The ZalaZONE test track contains an outer and an inner handling course section, for higher and lower speeds respectively. The total length of the high speed track is $2030 \mathrm{~m}$ with the pavement width of $12 \mathrm{~m}$. Here, the length of straight sections and the arc radius allow $120 \mathrm{~km} / \mathrm{h}$ to be reached. On the low speed handling track similar tests can be performed up to $60 \mathrm{~km} / \mathrm{h}$. Its overall length is $1330 \mathrm{~m}$ with the pavement width of $6 \mathrm{~m}$.

- Internal Road Network:

The primary role of the internal road network is to provide access to the various modules. At the same time, the internal road network of the test track is designed to meet road standards, allowing road tests and providing a connection between the elements such that the test vehicles can move from one element to the next without turning. This has an important role in the multi-element, longer cycle, special tests, even for autonomous vehicles.

- High Speed Oval:

The term high-speed oval means a closed course which, without significant deceleration, allows continuous driving in a given direction even at high speed. The proving ground has a high-speed oval track which has 
curved bends with a neutral speed. The High Speed Oval track enables the vehicle turning with released steering wheel at $200 \mathrm{~km} / \mathrm{h}$ due to the geometry of the pavement.

- Smart City Zone:

One of the uniqueness of the ZalaZONE test track is its built-in urban environment, called the Smart City Zone, which allows testing automated or fully autonomous vehicles in complex urban situations. The Smart City Zone includes different types of roads, intersections, and other features that are common in real-world urban area, e.g. bus stops, facades, traffic signs, or traffic lights. In addition to the listed items, bike paths, roundabouts, tram rails, low-adhesion surfaces, rainfall sections, and parking garages help the testing procedures.

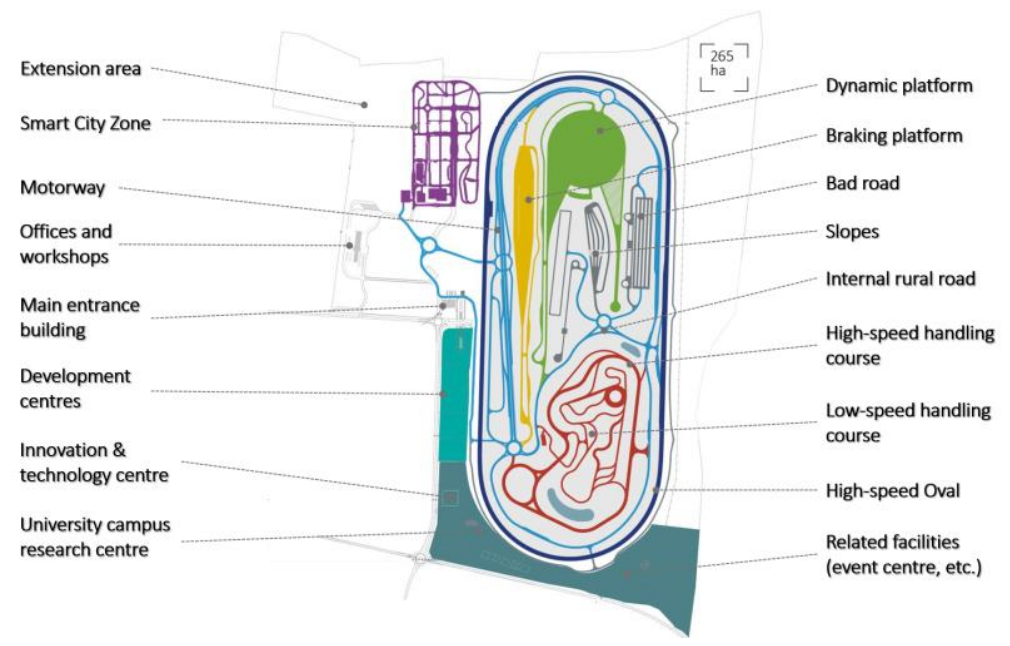

Figure 2. Main elements of ZalaZONE Proving Ground (AD in figure means Automated Driving)

\section{State-of-the-art simulation tools at ZalaZONE}

In ZalaZONE, currently, three state-of-the-art vehicle simulation software tools are in daily use. In the next paragraphs, three short summaries are provided based on the working experiences of the ZalaZONE virtual environment creation. 


\subsection{IPG CarMaker (Version 8.1) - IPG Automotive GmbH}

IPG CarMaker is an automotive simulation software of IPG Automotive $\mathrm{GmbH}$ (https://ipg-automotive.com/company/) specializing in passenger cars. The vehicle models available in it are characterized by sophisticated, all-round adjustments, and their vehicle dynamics models are efficient and accurate. Various tests can be performed using pre-set scenarios according to a measurement cycle set by a standardization authority. However, other methods, defined by the researcher for his own research purposes can also be used. It is possible to perform conventional vehicle dynamics tests even in the presence of the Advanced Driver Assistance System (ADAS). So-called "third-party" software (for example, MATLAB/Simulink, AVL Cruise, GT Suite, etc.) can also provide interfaces to refine the vehicle model and extend the modelling features that is also a very important functionality.

The environment building function is eligible to build simpler road network for standard tests, but it is possible to create more complex test environments by making some compromises. The so-called Scenario / Road module is used to create test environments. Newer versions of the software support the more and more popular and recognized OpenDRIVE ("de facto standard") format [15], which is widely used by the automotive industry to describe road networks.

The trajectories of the created road networks can be linked to real locations based on geographical latitude, longitude, and altitude, which can be simultaneously checked with an external map software and can even track vehicle movements during simulations.

\subsection{Prescan (version 2019) - Siemens PLM}

PreScan is another vehicle simulator software developed by Siemens PLM (https://www.plm.automation.siemens.com/global/en/products/sim center/prescan.html). PreScan is primarily desgined to test and simulate various sensors. In the secondary aspect, the vehicle dynamics used within the program is noteworthy. There are several sensor types of sensor models in the software and it is possible to use them as a real sensor, or an "idealized" one. Sensor settings, as well as vehicle parameterization and use of their functions, are available through the Matlab/SIMULINK software package.

The software is straightforward and user-friendly in the creation of an environment; it does not use a separate module; the central GUI is suitable for environment building. It has a relatively limited toolbar for creating road sections but contains unique elements (such as roundabouts) that are not available in the other software. A wide range of additional items can fit into the environment (e.g., trees, buildings, boards). Experience from modeling the ZalaZONE test track has shown 
that PreScan allows extremely easy track construction, even with a limited set of tools. By being compatible with Matlab/SIMULINK, there is plenty of Add-On available, allowing for a much more extensive range of testing and scenario building. Another advantage of the program is the simple trajectory drawing method. It is sufficient for a logically well-built road network to click on the connection points to draw the route.

PreScan can also work with other software (e.g. IPG CarMaker) using MATLAB/Simulink software package. It also allows for testing of built-in ADAS functions. It provides simulation capabilities in Model-in-the-Loop (MiL), SiL, and Hardware-in-the-Loop (HiL) environments with the ability to automate tests.

Another benefit of the software package is that its help guide is exceptionally userfriendly, describes many features with the use of the existing tools (e.g. Auto Emergency Braking (AEB) functions, creation of various ADAS scenarios).

\subsection{Vires Virtual Test Drive (version 2.2) - Hexagon, Vires Simulationtechnologie GmbH}

VTD Vires is also one of the most well-known automotive simulation software (https://vires.mscsoftware.com/). The software provides the ability to perform HiL, SiL, DiL (Driver-in-the-Loop), ViL tests. The software consists of two distinct parts: RoadDesigner and Scenario Editor. The program has the advantage of supporting OpenDrive, OpenCRG, and OpenScenario file formats, which are native formats of VTD Vires. It can likely be a strong basis for vehicle simulation software in the future [16]. It is possible to interface external software and modules to simulations such as Matlab/SIMULINK or other third-party software.

The RoadDesigner module allows the creation of different, well detailed virtual environments. With this tool, the virtual test environments can be more realistic, and it is easier to create a digital twin of a real test area based on the OpenDrive standard. With the Scenario Editor, one can create simulations of varying degrees of details to test basic vehicle and ADAS functionalities. Different sensor models can also be attached to the vehicle to extend the types of possible test cases with environment sensing simulations. Some of the Scenario parameters, such as weather, time, and time of day, can be changed in real-time when running simulations.

\section{Benchmark of automotive simulation software programs via modelling proving ground modules}

The main goal of the paper is to analyse and compare the three simulation software from a road modelling perspective. It is emphasized that rating of any of the investigated software is not planned because each has its advantages from a different 
point of views, i.e., in this paper, only the comprehensive experiences related to ZalaZONE Proving Ground modelling are provided

\subsection{Environment design basics in the various software}

In order to introduce the modelling capabilities of the software, a 'dummy' environment was created that contains all the typical road network elements. Hence, the created model includes a roundabout, straight sections with slopes, junction, turn, and a multilane section with entrance and exit lanes. The created modules are shown in Fig. 3 (a-IPG CarMaker, b-PreScan, c-VTD Vires).
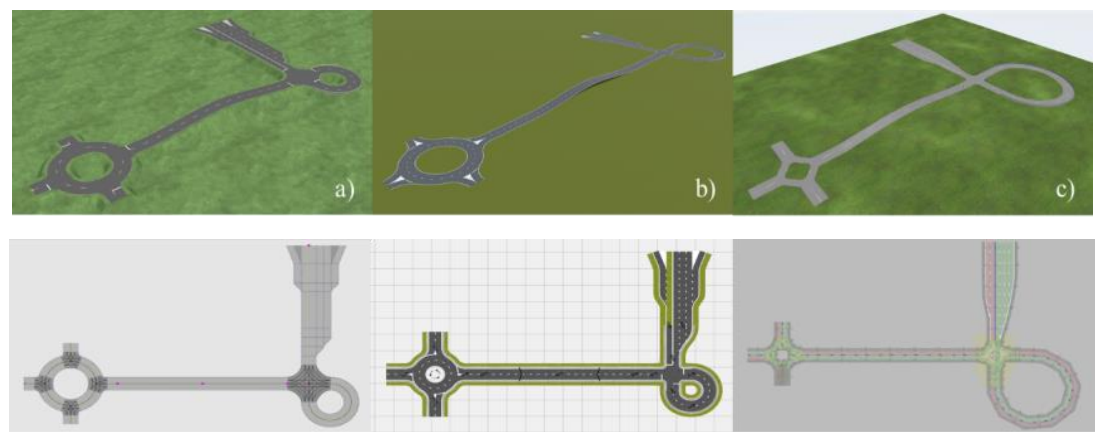

Figure 3. The dummy road-network in different software ( $a$-CarMaker, $b-$ PreScan, $c-V T D)$

In IPG CarMaker, one can find under the Parameters menu the Scenario/Road editor, which enables creating a new test environment. Road type can be selected easily from the menu, and then road drawing is straightforward. One can connect new road segments to the existing area, then parameterize them, and so on. There are more groups for the user like Road, Accessories, Scenery, Traffic, but the Road objects are the most important for the modelling. The Road objects group contains the following: Straight, Turn, Junction, Ram, Clothoid, Point list, Connect, and File. The last one provides the opportunity to import other file formats or existing road network segments.

Additional elements like Road marking, Traffic sign, Bridge, and Tunnel were also relevant for our work. After placing the Road objects, many more parameters can be set, e.g., the lanes' width or their lateral shift separately. More lanes can be also added to existing roads, and other features can be assigned to them afterward, like Elevation, Slope, and Camber profiles. The surfaces can be modified with the following elements: Beam, Cone, CRG, Friction, Mesh, Wave, or Lateral profile. Besides, additional elements can be added to each road segment, like a guard rail, lane markings, or textures for the different surfaces. Connecting two separate 
existing road sections, connect command has to be used, or the two roads must end at the same coordinates. In this case, there is the possibility of establishing the connection, even using a junction. The software does not include roundabouts, so these are built using junctions and turns. The dummy example road network can be built as described above. After the roundabout building, the straight road sections can be connected to it, and then the straight line elevation can be added using the Elevation profile. A junction can then be added to the existing road, and its two exits can be connected using turn roads. An expanding highway-type road can be connected to the last exit, which is turned into $2 \times 2$ lanes in each direction. The final steps are the placement of the guardrails and the road markings (e.g. dashed and continuous lines, double closing lines, perpendicular white lines for a give-way situation, etc.). The example model is illustrated in Fig. 3 -a).

In PreScan, it is possible to access the builder elements from the Library Elements menu within the GUI. Each element can be selected and moved to the drawing area with a Drag \& Drop method. Each item that is dropped in the Build Area gets a unique ID. PreScan contains several groups of objects (Environment, Infrastructure, Actors, Sensors, etc.), but the infrastructure and environment elements are the most important for the modelling.

The range of elements that can be used to road network building is quite broad, including the following elements: Bend Road, Curved Road (Bézier), Entrance Lane Road, Exit Lane Road, Flexible Road, Lane Adapter Road, Pedestrian Crossing, Ramp, Roundabout, Spiral Road (Clothoid), Straight Road, X Crossing, Y Crossing). Besides, there are several object groups (Road Markings, Buildings, Abstract Objects, Traffic Signs, Animated Elements, Reflectors \& Botts' dots, Generated Content, Other). However, from these, the Traffic Sign and Roadsign were the most relevant. Most objects can be parameterized in PreScan, and many other parameters can be set for path elements. In the case of a road section, different parameters can be adjusted including, the number of lanes, the curve radius, the width of the different paintings, the quality of the bench, if necessary, assigned to the road a sidewalk also can be added, which can also be parameterized. The speed limit for the road and the detectability by the built-in sensors also can be defined.

In the intersections (X Crossing, Y Crossing) and roundabouts, the properties, orientation, and parameters of the safety island can be set. Besides, guard rails, walls, or other texture can be added to each segment. In PreScan, the connection of the different roadblocks is simply by dragging the roads' connection points. It is also possible to connect the elements, but it is also possible to connect the lanes separately in some cases. In PreScan, slopes can be created using the Ramp element. The angle of the elevation can be defined by the length and height of the elevated section. One of PreScan's advantages is that it includes a predefined Roundabout element, so 
there is no need to build it separately. Lane Adapter is available to expand the lanes, but highway acceleration and exit lanes can also be used as separate elements. The dummy example road network can be built as described above.

After the roundabout is dropped in, the straight road sections can be connected to it, followed by the up and down ramp sections in the appropriate direction. To this, a junction can be added, and bend roads can connect its two exits.

To the last exit, a highway-type road with acceleration and exits lanes can be connected. The example file is illustrated in Fig. 2.

In VTD Vires, the first step of road and environment modelling is drawing the centreline as the road's base. The length and orientation of this road can be modified at this phase. This is not identified as a road network, but the sketch geometry can be turned into a path. VTD Vires is working with the OpenDRIVE file format since the path axis will be in the middle of the road. We can place lanes with any width on any side from this axis, which can be various types: driving, none driving, shoulder, bike, sidewalk, border, parking, etc. These different types perform different logical functions (For example, in a bike lane, a car cannot drive in traffic simulations.) The roads are connected using junctions. The roads' ends have to be selected for the connection, and then every possible driving path has to be defined separately.

VTD Vires does not contain a roundabout element, so this should be created using $\mathrm{T}$-junctions and small bend roads. Several small junctions can be connected with a larger one, which gives the final logical connection. Each lane can be assigned different materials, road markings, and additional environmental elements, such as a guardrail. Based on the above, the example road network can be easily built; this is illustrated in Fig. $3-\mathrm{c}$ ).

\subsection{Created proving ground modules}

Every module was created based on the blueprints (the original technical drawings). In VTD Vires and PreScan the blueprints could be used as underlays. A scaled blueprint of the test track was inserted on the right coordinate, and the roads were drawn on it. The underlay feature is only available from IPG CarMaker version 9.1, but the model was created in IPG CarMaker version 8.1.1. Therefore, the model is based on the accurate measurement of the blueprint.

In general, it is not possible to create a road network by hand that matches the blueprints completely, it serves as a basic layer, but with some compromise, the road network on the underlay can be well approximated. A general problem with modelling the test track accurately stems from the limitation of the different software. For example, cornering radii, special intersections, and unique test track elements, they require several overlapping layers. If the right logical connection for 
traffic simulation is required, application of additional layers is necessary. These layers are usually defined vertically on the same level, which can sometimes cause visualization issues, but this compromise is necessary to get a geometrically and logically correct road network. Thus, when preparing the test track, we tried to keep both geometrical and logical correctness, but in the case of certain elements, this could only be achieved with special solutions. The modules created in each software are listed below:

- High-speed Oval

- Dynamic Platform

- Braking Platform

- High-Speed Handling Course

- Low-Speed Handling Course

- Smart City Zone

- Motorway

- ADAS Surface

- Rural/Connection Roads

In this section, the Dynamic Platform and the Smart City Zone are described more verbosely as these test track elements have some specialties compared to conventional roads or race tracks. The Dynamic platform is a large flat surface with a few entry or exit connectors, and no traffic rules or routes prevail. The Smart City Zone on the other hand, is a typical "urban" environment but contains many unique or "tricky" intersections, roundabouts, and road sections.

\subsection{Modelling of the Smart City Zone}

In IPG CarMaker, the road network is based on the original blueprint of the test track. The model (Fig. 4 upper left) matches the real network with some minor deviations from the blueprint. Some differences are in the southern (figure 4 down middle) and northern section with a small roundabout and narrow T-junctions. These areas can affect the driving behaviour and sensor detection results, causing disparities in the simulations. To solve this problem, these sections are built with different road-width, and curve radius compared to the original blueprint. The software does not contain the special traffic signs of the ZalaZONE Proving Ground or Hungarian traffic signs. Some road signs could be found in the Japanese pack, but mainly third-party programs were used for the creation of the unique signs as shown in Figure 4 lower left. After the right scaling, these could be imported from image files. The road marking placement was simpler because every necessary road marking could be found in IPG's road designer as demonstrated in Figure 4 down right. Although the blueprint contains an elevation profile (Figure 4 upper right), it could not be accurately modelled. 


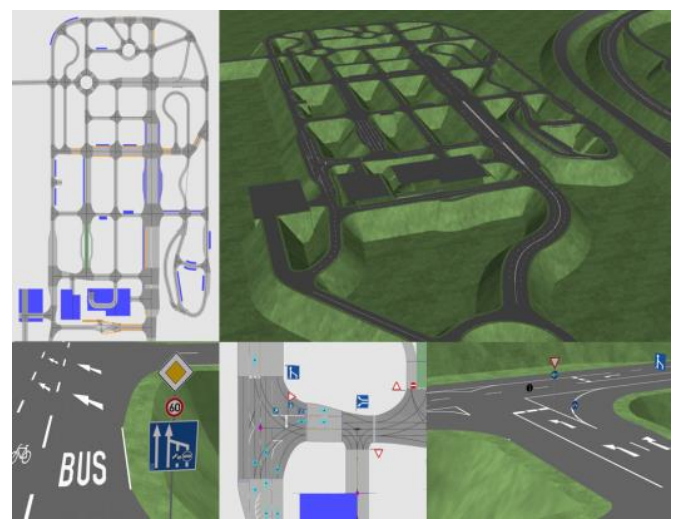

Figure 4. ZalaZONE Smart City Zone in IPG CarMaker

The built model in PreScan is logically correct, but there are geometrical differences from reality, mostly at junctions, where the program limits the parameters of the exit radius. Parking places are also planned in several locations, which could be created with the help of simple asphalt or concrete underlays. The most serious difficulties were in building the intersection on the western side shown in Figure 5, inside the Smart City module. The road markings are mostly included in the software, but the road signs set in the toolbox are incomplete for the given purpose. The track elements were built in $2 \mathrm{D}$; hence, the elevation profile is not defined.

In VTD Vires the roads are built very close to reality because the model is created using underlays (Fig. 6). The OpenDRIVE format is currently under development (standardization), hence some track sections cannot be correctly defined. The parking module is currently unavailable, so for the traffic simulations in the parking areas an invisible underlay must be created with normal roads. The appearance of the parking area can be built by increasing the border width and setting the material (pavement). While the large roundabout was built without difficulties, the small one is still under development due to the limitations of OpenDRIVE. The construction is logically correct, mostly. Scenarios can be run on $90 \%$ of the smart city zone (merging lanes are not quite right). The biggest problems are the creation of multilane junctions and roundabouts. The road markings are placed as planned, but some of the signs are incomplete. There is no elevation profile defined on the track elements. 


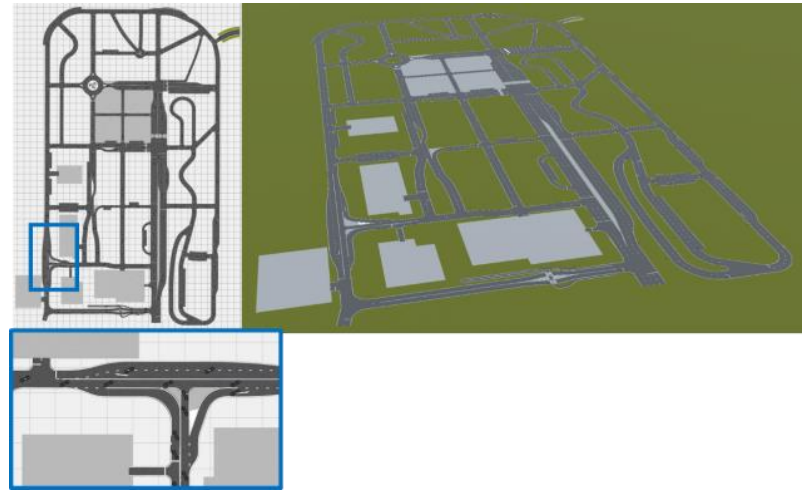

Figure 5. Smart City Zone in PreScan's GUI (upper left and right) and visualization tool with the most difficult junction (lower left)

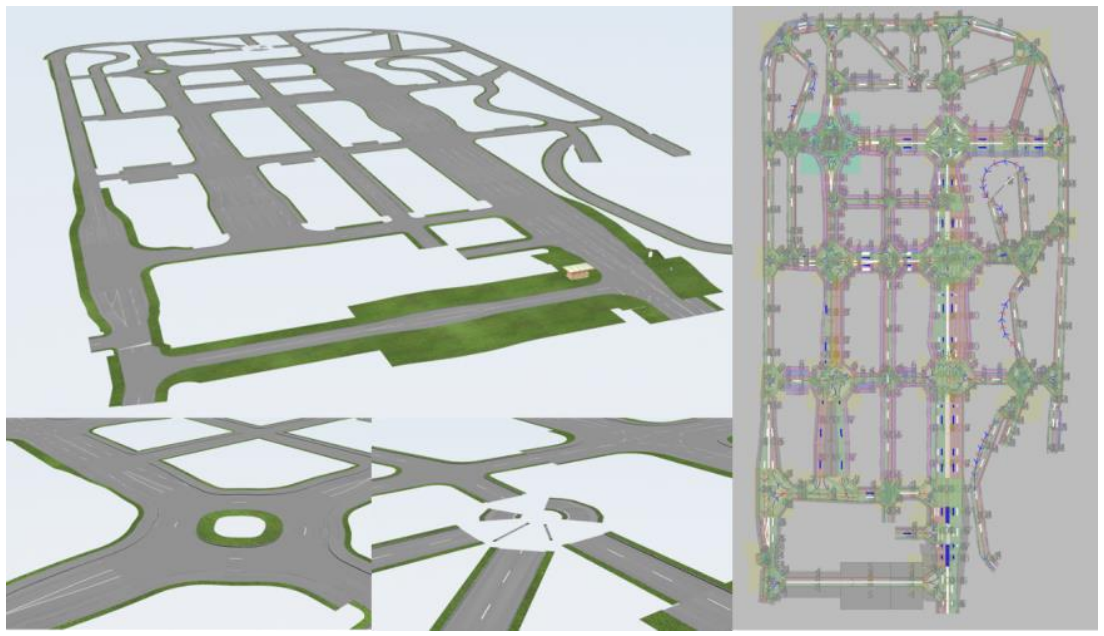

Figure 6. ZalaZONE Smart City Zone in VTD Vires

\subsection{Modelling of the Dynamic Platform}

The creation of the Dynamic platform (Fig. $7-a$ )) is a special task because the surface itself is also unique. Moreover, in IPG CarMaker the environment builder tool is not able to generate this full circle as a single road network element. First, an outer arc was built with the necessary junctions. Then, the internal lanes were created. These lanes are connected in one junction in the centre of the full circle and starting from here, and they become wider until they reach the outer arc, where they 
are connected with its junctions. They seem like circular-sectors. The triangleshaped connector parts of the acceleration lanes were built with similar methods. The ending sectors of these lanes have to be equal with the radius of the curves. (Fig. 7 - b)) In this case, the layers of the acceleration triangles cover the original circular sectors, but this is necessary for the right road network connection in order for the vehicles will be able to drive on the full dynamic platform (Fig. $7-$ c) d)).

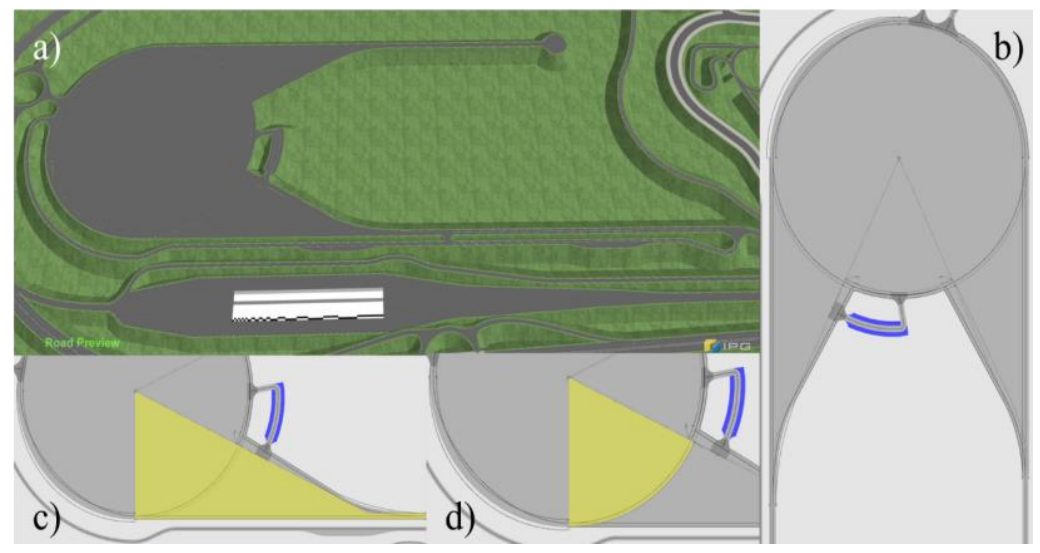

Figure 7. Dynamic platform in IPG CarMaker

PreScan contains the roundabout object, which made the building of the dynamic platform simpler (Hiba! A hivatkozási forrás nem található.). For the full circle, two roundabouts are needed because one roundabout is able to handle only ten different lanes. The outer roundabout has all of the exit lanes, which are necessary for the junctions of the platform (like the connectors of the turn-back lane and the parking area). In this software, only just the existing acceleration lane was created. In this case, a similar method was applied as in IPG CarMaker, because the acceleration triangle is an overlay that partially covers the outer roundabout. PreScan is not sensitive to the missing road connections, but one connected lane was defined between the acceleration lane and the turn-back lane for traffic simulation purposes. 


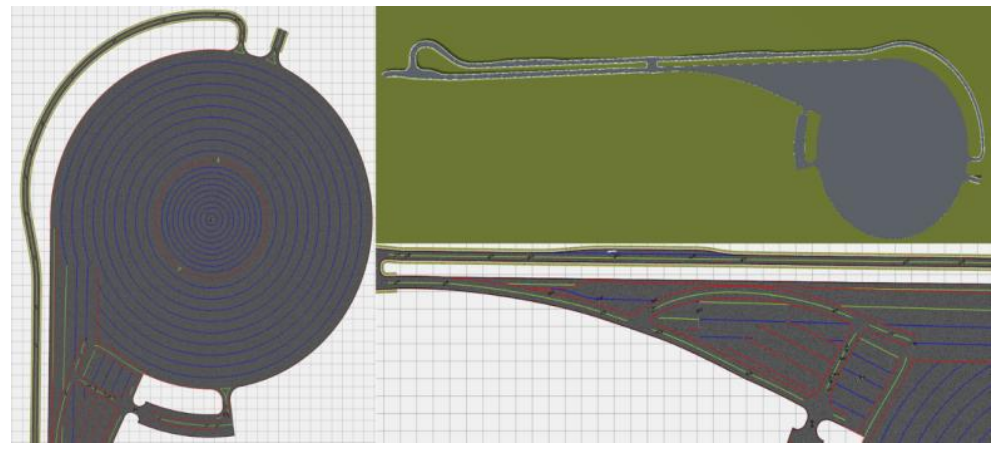

Figure 8. The PreScan model of the Dynamic Platform in the GUI and in the visualization tool

In Vires VTD, two different layers were created (Fig. 9). The first layer (Fig. 9 down right) contains all the junctions and necessary road connectors and provides the right road-network logic for the traffic simulation, but this layer is hidden in the visualization. The second layer (Fig. 9 left side) is responsible for the right visual appearance. This could be drawn easily in this software. However, this object itself is not identified as a road object or road-network. The final result is shown by Fig. 9 (upper right).

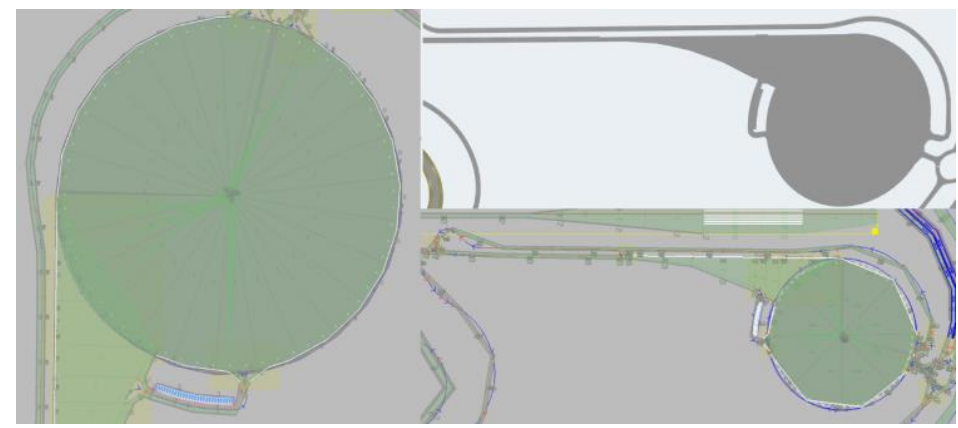

Figure 9. VTD model of the Dynamic platform in ROD editor and in the simulations

\section{Conclusion}

In this paper, state-of-the-art simulation software tools were presented, in which the ZalaZONE proving ground models have been built up for scientific research and practical vehicle pre-testing purposes. Through this work presented, advantages, different features, even some limitations and novel, proposed model building techniques of the different simulation tools were identified. It is important to note 
that all of the considered software could have advantages or disadvantages. It ust be noted that the presented work did not aim to prioritize any of the software tools but to present the relevant features and provide useful suggestions concerning scientific or practical application. The strengths and weaknesses of the tools basically depend on the specific usage. It was clearly observable that these tools are not developed for the creation of road networks with such large size and complexity as the ZalaZONE Proving Ground has. Some software can handle this easier, or provide the opportunity to save the whole model in separate files.

It can be noted in general that the creation of a road-network that can match the original plans geometrically in the whole area is only possible by using multilayers that cover each other. It does not provide the right logical connections in the roadnetwork; hence usually, an additional layer with the right connections is also necessary for the software. Moreover, the models of the investigated tools also have difficulties with special elevation attributes, e.g., banked curves, slopes, etc.

When the construction of ZalaZONE is finished, a laser scanning about the whole area is be carried out to provide a more accurate digital copy of the real environment. Based on this future data set, OpenDRIVE network is also planned to be generated, which then will be imported smoothly into any modelling software. (via the modelling the ZalaZONE Proving Ground of Hungary)

As a continuous result of the research of proving ground modelling, an open access repository has been started on GitHub where the models are freely available in different file formats for simulation software IPG CarMaker, PreScan, VTD Vires: https://github.com/BMEAutomatedDrive [16], [17]. The more, this repository contains two additional models created in SUMO and Unity $3 D$ which were not investigated in the paper as they are not specific automotive programs. SUMO (Simulation of Urban MObility) [18] is a free and open microscopic road traffic simulation suite. Unity $3 D$ [19] is a graphical game engine for realistic 3D visualization. As another future work the modelling works of ZalaZONE is planned within Matlab/Simulink (Automated Driving Toolbox) being a generic tool for vehicle engineers.

\section{Acknowledgments}

The research reported in this paper was supported by the Higher Education Excellence Program of the Ministry of Human Capacities in the frame of Artificial Intelligence research area of Budapest University of Technology and Economics (BME FIKP-MI/FM). The project has also been supported by the European Union, co-financed by the European Social Fund. EFOP-3.6.2-16-2017-00002. 


\section{References}

[1] T. Tettamanti, I. Varga, Zs. Szalay, Impacts of Autonomous Cars from a Traffic Engineering Perspective, Periodica Polytechnica Transportation Engineering 44 (4) (2016), pp. 244-250.

doi: https://doi.org/10.3311/PPtr. 9464

[2] P. Koopman and M. Wagner, Challenges in Autonomous Vehicle Testing and Validation. SAE Int. J. Trans. Safety 4 (1) (2016), pp. 15-24.

[3] Y. Kang, H. Yin, C. Berger, Test Your Self-Driving Algorithm: An Overview of Publicly Available Driving Datasets and Virtual Testing Environments, IEEE Transactions on Intelligent Vehicles, 4 (2) (2019), pp. 171-185. doi: https://doi.org/10.1109/TIV.2018.2886678

[4] S. Riedmaier, J. Nesensohn, et al., Validation of X-in-the-Loop Approaches for Virtual Homologation of Automated Driving Functions. In: Proceedings of the $11^{\text {th }}$ Graz Symposium Virtual Vehicle (2018).

[5] Y. Laschinsky, K. von Neumann-Cosel, et al., Evaluation of an Active Safety Light using Virtual Test Drive within Vehicle in the Loop, IEEE International Conference on Industrial Technology, Vina del Mar, (2010), pp. 1119-1112.

doi: https://doi.org/10.1109/ICIT.2010.5472583

[6] R. Kallweit, P. Prescher, M. Butenuth, Vehicle-in-the-loop: augmenting realworld driving tests with virtual scenarios in order to enhance validation of active safety systems. 25th International Technical Conference on the Enhanced Safety of Vehicles (ESV), Detroit (2017).

[7] D. Gruyer, S. Demmel, et al., Simulation architecture for the design of Cooperative Collision Warning systems. 2012 15th International IEEE Conference on Intelligent Transportation Systems, (2012), pp. 697-703. doi: https://doi.org/10.1109/ITSC.2012.6338733

[8] A. Belbachir, J-C. Smal, J-M. Blosseville, D. Gruyer, Simulation-Driven Validation of Advanced Driving-Assistance Systems, Procedia - Social and Behavioral Sciences, 48, (2012), 1205-1214, ISSN 1877-0428. doi: https://doi.org/10.1016/j.sbspro.2012.06.1096 
[9] M. Worm, R. van der Made, Verifying ADAS and autonomous driving performance, Siemens PLM Software White paper (2019).

[10] H. Németh, A. Háry, et al., Proving Ground Test Scenarios in Mixed Virtual and Real Environment for Highly Automated Driving. In: Proff H. (eds) Mobilität in Zeiten der Veränderung. Springer Gabler, Wiesbaden (2019), pp. 199-210.

doi: https://doi.org/10.1007/978-3-658-26107-8_15

[11] Zs. Szalay, M. Szalai, et al., Proof of concept for Scenario-in-the-Loop (SciL) testing for autonomous vehicle technology, Graz: IEEE International Conference on Connected Vehicles and Expo (ICCVE), (2019), pp. 1-5. doi: https://doi.org/10.1109/ICCVE45908.2019.8965086

[12] Zs. Szalay, Z. Hamar, P. Simon, A Multi-layer Autonomous Vehicle and Simulation Validation Ecosystem Axis: ZalaZONE. In: Strand M., Dillmann R., Menegatti E., Ghidoni S. (eds) Intelligent Autonomous Systems 15. IAS 2018. Advances in Intelligent Systems and Computing, Vol 867, Springer, Cham, 2019. doi: https://doi.org/10.1007/978-3-030-01370-7_74

[13] Zs. Szalay, T. Tettamanti, et al., Development of a Test Track for Driverless Cars: Vehicle Design, Track Configuration, and Liability Considerations, Periodica Polytechnica Transportation Engineering, 46 (1) (2018), pp. 29 35.

doi: https://doi.org/10.3311/PPtr.10753

[14] Zs. Szalay, Á. Nyerges, Z. Hamar, M. Hesz, Technical Specification Methodology for an Automotive Proving Ground Dedicated to Connected and Automated Vehicles, Periodica Polytechnica Transportation Engineering, 45 (3) (2017), pp. 168-174.

doi: https://doi.org/10.3311/PPtr.10708

[15] M. Dupuis and H. Grezlikowski, OpenDRIVE® - an open standard for the description of roads in driving simulations. In Driving Simulation Conference (2006), pp. 25-36, Paris, France.

[16] BME AutomatedDrive github repository

URL https://github.com/BMEAutomatedDrive 
[17] BMEAutomatedDrive homepage

URL https://www.automateddrive.bme.hu/

[18] P. A. Lopez et al., Microscopic Traffic Simulation using SUMO, 2018 21st International Conference on Intelligent Transportation Systems (ITSC), Maui, HI, (2018), pp. 2575-2582.

doi: https://doi.org/10.1109/ITSC.2018.8569938

[19] M. Szalai, B. Varga, T. Tettamanti and V. Tihanyi, Mixed reality test environment for autonomous cars using Unity 3D and SUMO, 2020 IEEE 18th World Symposium on Applied Machine Intelligence and Informatics (SAMI), Herlany, Slovakia, (2020), pp. 73-78.

doi: https://doi.org/10.1109/SAMI48414.2020.9108745 